Proc. Indian Acad. Sci., Vol. 87 A (E \& P Sciences-4), No. 11, November 1978, pp. 263-270, (c) printed in India

\title{
Measurement of apparent velocity and azimuth of seismic signals recorded at an array
}

\author{
A RAM \\ Department of Geophysics, Banaras Hindu University, Varanasi 221005
}

MS received 3 December 1977; revised 24 June 1978

\begin{abstract}
A set of computer programs was written which enables one to determine automatically, the apparent velocity and azimuth of any portion of the seismic wavetrain recorded at various seismic arrays. All that is required to use these programs is that the raw data be available on digital tape and that the user have access to any modern computer. The programs are written in Fortran IV and make use of the adaptive processing method whereby the arrival times of the wavefront at each sensor are accurately determined by cross-correlating the observed wavelet of interest with the corresponding wave on the beam trace. The new arrival times are then used to create a new and improved beam and the whole operation is repeated in an iterative manner until convergence takes place.
\end{abstract}

Keywords. Apparent velocity; azimuth; seismic signals; array.

\section{Introduction}

The most significant advantage of a seismic array over a single seismic station is that the slowness (or slope of the travel-time graph), apparent velocity and azimuths can be measured directly from the observations. Theie measurements are made in addition to the usual measurements of travel-time, amplitude and spectra. The direct measurements of the slope of the travel-time curve as a function of distance can then be used to invert the observations to obtain a velocity-depth curve. Because transition zones, discontinuities, lateral inhomogeneities, etc. may cause multipathing of the seismic energy it is essential that in any detailed study of earth structure, slowness and azimuth measurements must be made over several seconds of the seismic wavetrain after the initial onset. Furthermore because of the great complexities in earth structure and complicated source functions which are usually produced by an earthquake, as many observations as possible should be made in order that statistically significant results can be obtained. The manual method of 'picking' relative arrival times at each sensor from a set of array traces is not only tedious but also does not make full use of the shape of the waveform and hence was not considered by the author. It is therefore necessary that digital methods involving the use of a computer be employed.

In this paper a computer program package is described which enables a user to determine automatically the slowness, apparent velocity and aximuth of any portion of the seismic wavetrain recorded at various seismic arrays. All that is required to use these programs is that the raw data be available on a digital tape which can be 
read by any modern computer. The programs are written in Fortran IV and make use of the adaptive processing method whereby the arrival time of the wavefront at each sensor is accurately determined by cross-correlating the observed wavelet of interest with the corresponding wave on the beam trace. The new arrival times are then used to create a new and improved beam and the whole operation is repeated in an iterative manner until convergence takes place. Descriptions and evaluations of the method are given by Gangi and Fairborn (1968), Farrell (1971), Bungum and Husebye (1971), King et al (1973), and Mereu and Ram (1975). Apart from the fact that full use is made of the shape of the wavelet to determine accurate values of apparent velocity and azimuth, the method has also an advantage that it can readily be programmed for any moderately sized computer.

\section{Program package for seismic data analysis}

The computer program package, which was developed, is based on the adaptive processing technique. The method is adaptive in that the delay values resulting from each iteration are used to provide a refined reference signal for the subsequent iteration. The programs were designed primarily for the UKAEA-type medium aperture arrays which have 2 lines called red and blue lines. Should the data come from another type of array, these programs may still be applied without any modification provided the user divides the channels into two groups. This division may be made arbitrarily and should not affect the final answers. The programs were written to handle 36 see of 20 channel data. If core size is a problem $(<30 \mathrm{~K})$, modifications can easily be made to reduce the amount of data read into the core so that shorter lengths of record (for example $6 \mathrm{sec}$ ) are processed at a time. The whole process is then repeated several times to cover the time period of interest. The programs and subroutines for the adaptive processing techniques were described by Mereu and Ram (1975) and are a rewrite and modifications of the ones written by King et al (1973). Those programs were locked into the Australian National University data processing system and were not readily usable outside that system. This package of programs has also attracted the interest of a number of the array seismologists at the Department of Energy, Mines and Resources in Ottawa and at the University of Oxford in England. They have now incorporated these programs into their own research projects.

The computational steps which were taken in the program for this method are given in table 1. The main control parameters and different subroutines required for processing of array data are illustrated in tables 2 and 3 respectively.

Through the use of control cards the user can control the number of measurements (NMEAS) to be made over a coherent wavelet, the size of the windows (WF1, WF2) to be used and the position relative to the centre of the wavelet at which he would like measurements to be made (KBGN). With these options he is able to make measurements over each individual peak and trough separately or if he wishes he may use the whole wavelet at once. He can also make as many measurements as he would like along the coherent event. From a number of preliminary experiments (Ram 1976), it was found that three to five measurements are usually sufficient, the first using the onset of the wavelet, and the last using the tail end of the wavelet with the other measurements overlapping the first and last as shown in Figure 1a. 
Table 1. Major computational steps for the measurement of slowness, apparent velocity and azimuth using the adaptive processing programs

1. Select event, digitise and store on magnetic tape/disk.

2. Input e.g. SR, expected AZ, slowness, window lengths, filter limits, number of iterations, station co-ordinates, etc.

3. Data are bandpass filtered.

4. Calculate initial steering delays.

5. Form Time Averaged Product (TAP) and initial beam (SRB).

6. Select all wavelets to be measured automatically from TAP trace.

7. For each channel: subtract the data of that channel from the beam, evaluate the cross correlation of that channel and the depleted beam, locate the maximum value of the correlation function. The channel is then added back into the beam realigned.

8. Further refine the revised time delays by using a parabolic interpolation.

9. Obtain a solution of the normal equations using the refined time delays (Kelly 1964 and Otsuka 1966).

10. Compare the solution to check for convergence, form new beam, repeat 7-10 until convergence takes place.

11. Plot and label the records and solutions, print the results together with punched cards and storage on magnetic tape.

The method is adaptive in that the delay values resulting from each iteration are used to provide a refined reference signal for the subsequent iteration.
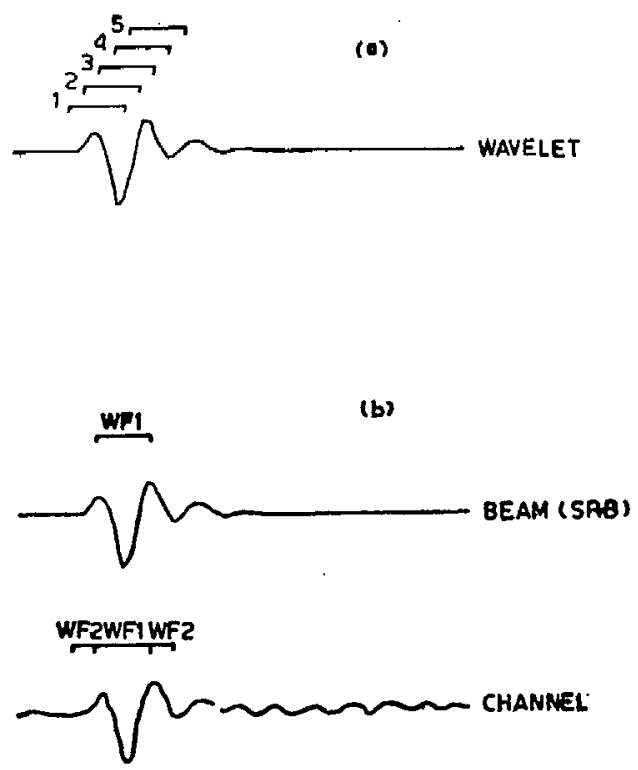

Figure 1 a. Diagram showing how the five measurements are made at different equally spaced locations along a wavelet and $1 \mathrm{~b}$. Shows the window sizes normal[y used in the cross-correlation procedure (WF $1=0.75 \mathrm{sec}$ and WF2 $=0.34 \mathrm{sec}$.).

The control parameters to set the size of the windows to be used in the cross-correlation procedure are WF1 and WF2. Their use when applied to a portion of a wavelet is illustrated in figure $1 \mathrm{~b}$. The beam signal of width WF1 is cross-correlated with the channel trace of width WF2+WF1+WF2. The position of the maximum value of this cross-correlation function is used as the relative arrival time position of the wave. Care must be taken not to choose WF2 too large as then the correlation functions 
may have two maxima and the computer may choose the wrong one for the arrival time position. If by chance the maxima occur at the end points of the comelation function they are rejected as not representing the true arrival time positions. A parabolic fit (subroutine TPARFT) is also made on the three largest values of the correlation function to refine its maximum position.

Aliasing is a problem which can occur when the computer fails to align corresponding peaks on successive traces. Tests with synthetic data showed (Ram 1976) that this tends to occur when the initial trial velocity differs from the true velocity by more than $3 \mathrm{~km} / \mathrm{sec}$ or the azimuth by more than $8^{\circ}$. The tests also showed that when this occurs convergence usually fails to take place of the standard-errors were

Table 2. Main control parameters

\begin{tabular}{|c|c|c|}
\hline $\begin{array}{l}\text { Control } \\
\text { parameter }\end{array}$ & Description & $\begin{array}{c}\text { Typical } \\
\text { value }\end{array}$ \\
\hline SR & Sampling rate per sec. & 20 \\
\hline NPT & Number of data points per channel & 720 \\
\hline NCH & Number of channels & 20 \\
\hline TAPWIND & Window size for time average product (TAP & $0.75 \mathrm{sec}$ \\
\hline WNDEV & Window size for event subroutine & $2.0 \mathrm{sec}$ \\
\hline METHEV & $\begin{array}{l}\text { Method of choosing coherent events along trace } \\
\text { If METHEV =1, computer finds coherent events for user } 1 \\
\text { If METHEV }=2 \text {, user selects his own coherent events } \\
\text { If METHEV }=3 \text {, computer performs calculations along whole } \\
\text { trace }\end{array}$ & \\
\hline KBGN & Starting point relative to event of interest & 10 units \\
\hline TLEV & Duration of an event & $1 \mathrm{sec}$ \\
\hline NMEAS & Number of measurements per event & 3 to 5 \\
\hline HT & $\mathbf{H T}=$ Height of characters to be plotted & \\
\hline NCHMN & Minimum number of channels to be plotted & 1 \\
\hline MCHMX & $\begin{array}{l}\text { Maximum number of channels to be plotted } \\
\text { (NCHMX }=\mathrm{NCH}+\text { time channel) }\end{array}$ & 21 \\
\hline GIVSL & Given value to plot slowness axis & $11.0 \mathrm{sec} / \mathrm{deg}$ \\
\hline GIVVB & Given value for apparent velocity & $10.0 \mathrm{~km} / \mathrm{sec}$ \\
\hline GIVAZ & Starting value for azimuth $=$ expected azimuth & \\
\hline TL & Desired length of band pass filter & $3.0 \mathrm{sec}$ \\
\hline FL & Low pass filter frequency & 3.0 Hz \\
\hline FH & High pass filter frequency & $0.4 \mathrm{~Hz}$ \\
\hline WF1 & Window size for beam (SRB) & $0.75 \mathrm{sec}$ \\
\hline WF2 & Window size for channel traces & $0.34 \mathrm{sec}$ \\
\hline ALEX & $\begin{array}{l}\text { Correlation limit value } \\
\text { If the average correlation is less than } \\
\text { ALEX no answer will be plotted }\end{array}$ & 0.90 \\
\hline $\left.\begin{array}{l}\text { JUP1 } \\
\text { JUP2 }\end{array}\right\}$ & $\begin{array}{l}\text { Options to reduce the number of points } \\
\text { used to determine the maximum of the } \\
\text { correlation function }\end{array}$ & $\begin{array}{l}4 \\
4\end{array}$ \\
\hline KITL & Maximum number of iterations & 6 \\
\hline NPUNCH & Gives an option to punch out the results on cards & \\
\hline NWT & Gives an option to write the results on magnetic tape & \\
\hline
\end{tabular}


Table 3. List of subroutines required for the adaptive processing package*

Subroutines
Function

exceedingly large. These solutions were rejected automatically by the computer using present levels of the correlation coefficient.

The following three normal equations as described by Kelly (1964) and Otsuka (1966) are solved using the refined time delays:

$$
\sum_{i=1}^{N} X_{i}\left(X_{t} P+Y_{\imath} Q+T_{t}-T_{0}\right)=0
$$

Proc. A. -7 


$$
\begin{aligned}
& \sum_{i=1}^{N} Y_{i}\left(X_{i} P+Y_{i} Q+T_{i}-T_{0}\right)=0 \\
& \text { and } \quad \sum_{i=1}^{N}\left(X_{i} P+Y_{i} Q+T_{i}-T_{0}\right)_{i}^{\prime}=0
\end{aligned}
$$

where $\left(X_{i}, Y_{t}\right)$ are array coordinates; $T_{i}$ is arrival time of wavefront at the $i$ th seismometer as determined by the correlation maximum and $T_{0}$ the time at the origin (or cross-over point of the array)

$$
\begin{aligned}
& P=\sin \phi / V, \\
& Q=\cos \phi / V .
\end{aligned}
$$

The apparent velocity $(V)$, slowness $(d T / d \triangle)$ and apparent azimuth $(\phi)$ are given by

$$
V=\left(P^{2}+Q^{2}\right)^{-1 / 2}
$$

$$
\text { Slowness }(d T / d \triangle)=\frac{r_{0}}{V}=r_{0}\left(P^{2}+Q^{2}\right)^{1 / 2} \text {, }
$$

and $\quad \tan \phi=P / Q$;

䭪 279

D 19 TEAR

OAJ G. TIRE

H $M$ SEL

102411.1

AARI. TIIIEE

H. $A$ SEC

10 2922.1

LOCATION

$30.284 \mathrm{~N}$

$73.980 E$

$T=31\} .00$ SEC RT $=35.57$ SEC OELTA=24.77 $P H] \times 353.5$ FD= $130 \mathrm{KH}$ $\mathrm{MB}=5.1$

MAND PASS IL-3.0 HZ $\mathrm{FH}=0.4 \mathrm{H} 2$

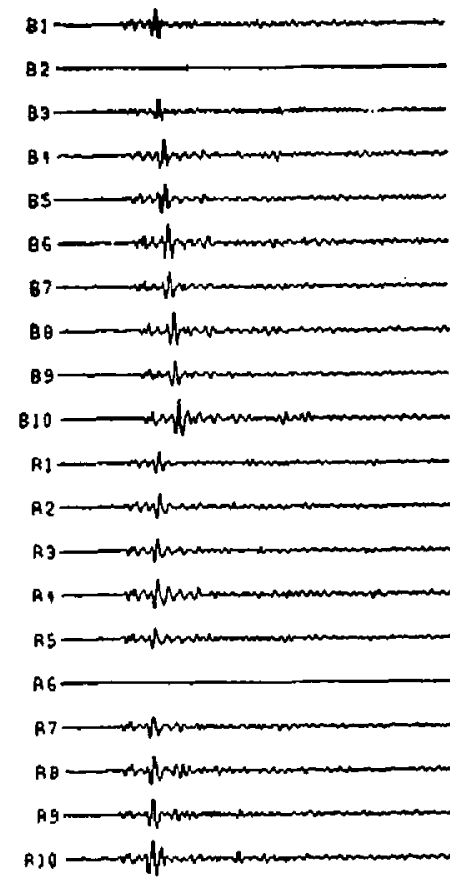

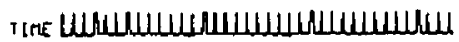

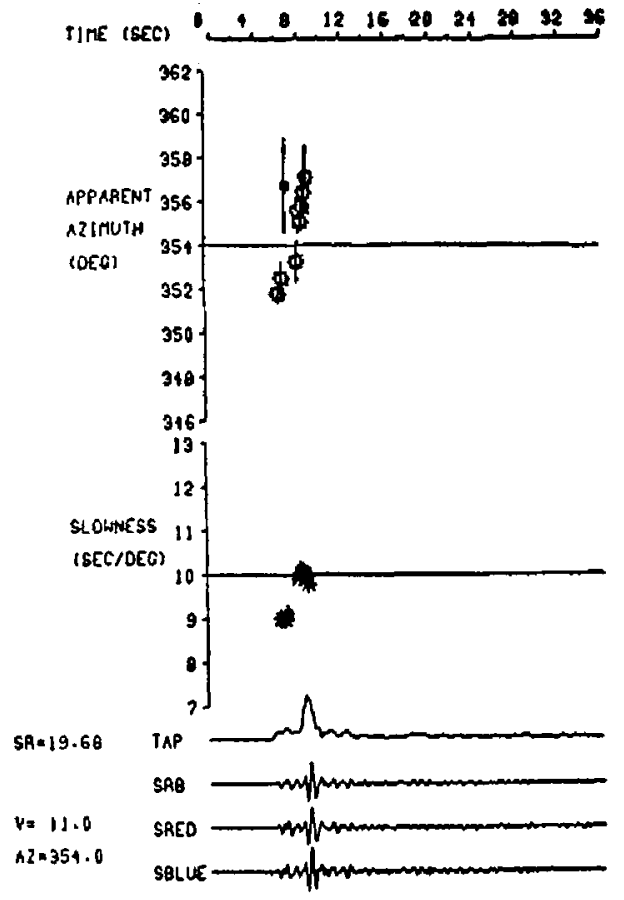

Figure 2. Earthquake recorded at the Gauribidanur Seismic array (GBA), $\triangle c=25.72^{\circ}$, Azimuth $=353.5^{\circ}(\mathrm{T}=$ total travel time, $R T=$ reduced travel time, $\mathrm{V}$ and $\mathrm{AZ}$ are the given apparent velocity and azimuth used to form the initial beam (SRB) and corresponding adaptive processed solution). 

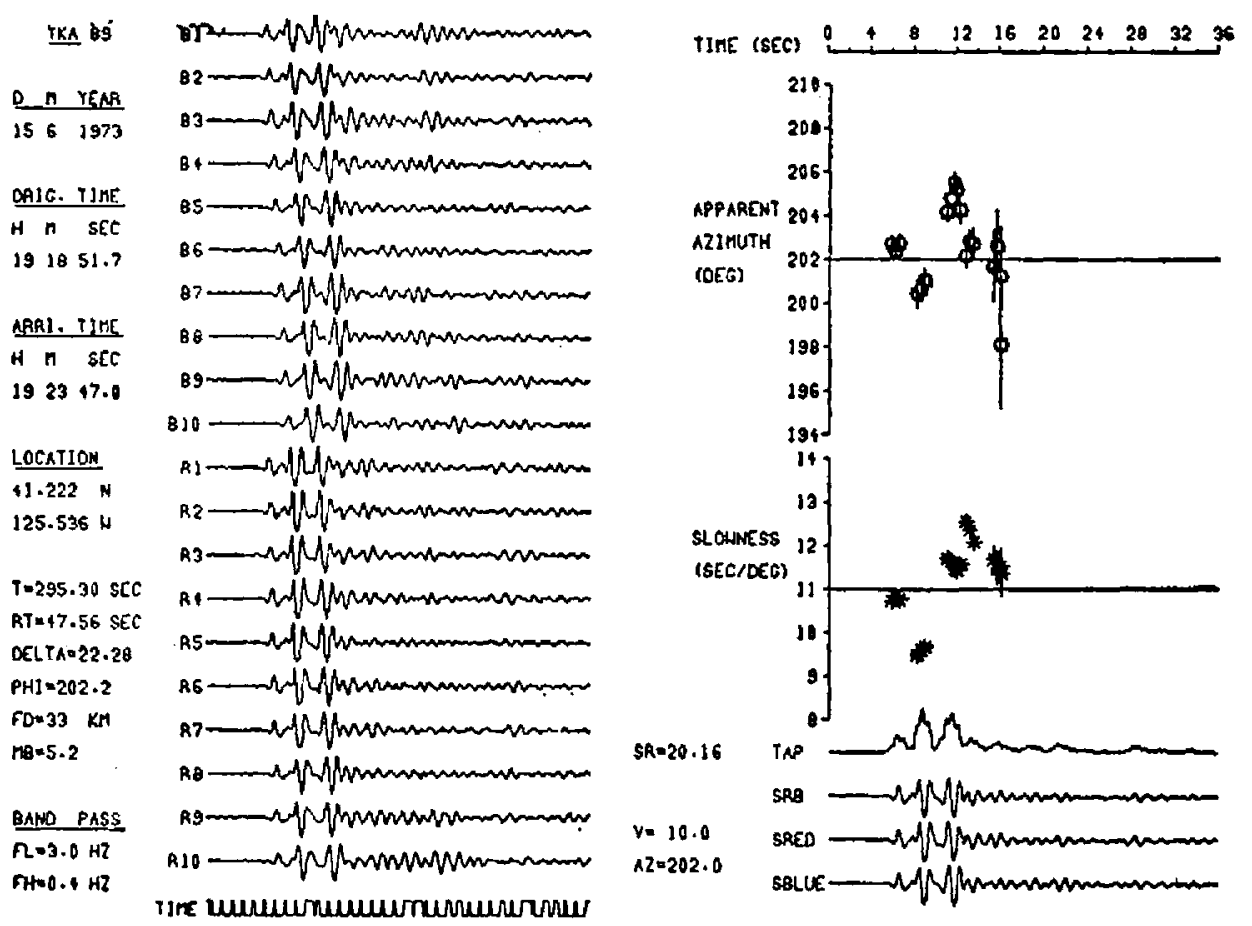

Figure 3. Earthquake recorded at the Yellowknife seismic array (YKA), $\Delta c=22.52^{\circ}$, Azimuth $=202 \cdot 2^{\circ}(T=$ total travel time, $R T=$ reduced travel time, $V$ and $A Z$ are the given apparent velocity and azimuth used to form the initial beam (SRB) and corresponding adaptive processed solution).

$r_{0}$ is radius of the earth and $N$ represents total number of seismometers in an array.

After solving these equations, the solution is checked for convergence. A new and improved beam is formed and the whole operation is repeated (see table 1) until convergence takes place. The results are printed, plotted and written on magnetic tape for further analysis.

\section{Examples}

The examples shown in figures 2 and 3 are solutions of the events recorded at the Gauribidanur Seismic Array (India) and the Yellowknife Seismic Array (Canada). These events were processed by the CDC CYBER 73 computer at the University of Western Ontario, London, Canada. The total computing time for each earthquake was approximately $90 \mathrm{sec}$ (or about $3 \mathrm{sec}$ for each data point). This includes the time spent in reading the data from the digital tape, filtering, analysing and finally storing the results on a magnetic tape. The total plotting time for the results of each earthquake was approximately $10 \mathrm{~min}$. The error bars are those obtained from the least squares solution. The size of the points used to display the answers is a measure of how well the signals correlated across the array. 


\section{Acknowledgements}

This research was carried out at the University of Western Ontario, Canada. The author is very grateful to Prof. R F Mereu for his continued support and supervision in this project. The financial assistance by (i) National Research Council of Canada grant No. A-1793 and (ii) the Canadian Commonwealth Scholarship and Fellowship Plan is thankfully acknowledged.

\section{References}

Bungum H and Husebye E S 1971 Pure Appl. Geophys. 9156

Farrell E J 1971 Geophysics 36822

Gangi A F and Fairborn J W 1968 Suppl. Nwovo. Cim. Ser. 16105

Kelley E J 1964 M I T Group Rep. 44

King D W, Mereu R F and Muirhead K J 1973 Geophys. J. R. Astron. Soc. 35137

Mereu R F and Ram A 1975 in Exploitation of seismograph networks ed K G Beauchamp (NATO Adv. Study Inst. Series) p. 327

Otsuka M 1966 Bull. Seism. Soc, Am. 56223

Ram A 1976 The identification and interpretation of upper mantle travel time branches from slowness measurements made on data recorded at the Gauribidanur (India) and Yellowknife (Canada) seismic arrays, Ph.D. thesis (University of Western Ontario, Canada) 